\title{
(2) \\ OPEN ACCESS \\ Misdiagnosis of prolonged psychogenic non-epileptic seizures as status epilepticus: epidemiology and associated risks
}

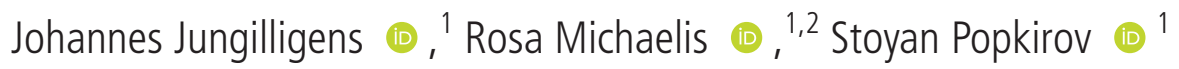

- Additional supplemental material is published online only. To view, please visit the journal online (http://dx. doi.org/10.1136/jnnp-2021 326443).

${ }^{1}$ Department of Neurology, University Hospital Knappschaftskrankenhaus Bochum, Ruhr University Bochum, Bochum, Germany ${ }^{2}$ Department of Neurology, Gemeinschaftskrankenhaus Herdecke, University of Witten/ Herdecke, Witten, Germany

\section{Correspondence to} Dr Johannes Jungilligens, Department of Neurology, University Hospital Knappschaftskrankenhaus Bochum, Ruhr University Bochum, 44892 Bochum, Germany; johannes. jungilligens@rub.de

Received 19 February 2021 Accepted 21 July 2021 Published Online First 6 August 202

Check for updates

(C) Author(s) (or their employer(s)) 2021. Re-use permitted under CC BY-NC. No commercial re-use. See rights and permissions. Published by BMJ.

To cite: Jungilligens J, Michaelis R, Popkirov S. $J$ Neurol Neurosurg Psychiatry 2021:92:1341-1345.

\begin{abstract}
Objective To determine the epidemiology of prolonged psychogenic non-epileptic seizures (pPNES) misdiagnosed as status epilepticus, as well as the risks associated with non-indicated treatment.

Methods We performed an individual patient data analysis from the Rapid Anticonvulsant Medication Prior to Arrival Trial (RAMPART) and the Established Status Epilepticus Treatment Trial (ESETT) to assess incidence, patient characteristics and clinical course of misdiagnosed pPNES.
\end{abstract}

Results Among 980 patients aged 8 years or older diagnosed and treated for status epilepticus in RAMPART and ESETT, 79 (8.1\%) were discharged with a final diagnosis of pPNES. The relative incidence was highest in adolescents and young adults (20.1\%). The typical female preponderance seen in that age bracket was not evident in children and older adults. Adverse effects, including respiratory depression and intubation, were documented in $26 \%$ of patients with pPNES receiving benzodiazepines in RAMPART and $33 \%$ of patients receiving additional second-line medication in ESETT. In ESETT, patients who were treated with benzodiazepines before hospital admission had higher rates of unresponsiveness and severe adverse effects than those treated after admission, suggesting cumulative effects of accelerated treatment momentum. Across trials, one in five patients with pPNES were admitted to an intensive care unit.

Conclusions Misdiagnosis and treatment of pPNES as status epilepticus are a common and widespread problem with deleterious consequences. Mitigating it will require training of emergency staff in semiological diagnosis. Status epilepticus response protocols should incorporate appropriate diagnostic re-evaluations at each step of treatment escalation, especially in clinical trials.

\section{INTRODUCTION}

Status epilepticus is thought to cause irreversible harm after more than 30 min of ongoing convulsions, so early aggressive treatment is recommended by most guidelines. ${ }^{1}$ Within minutes, benzodiazepines should be administered as first-line medication, with high-dose antiepileptic drugs and intubation as second-line and third-line treatment in cases of refractory seizure activity. This compression of treatment timeframes has proven beneficial for the majority of patients with status epilepticus. ${ }^{2}$ However, this treatment imperative can come at the cost of diagnostic accuracy in emergencies.
The main differential to consider when assessing patients with suspected (convulsive) status epilepticus are dissociative seizures, also known as functional or psychogenic non-epileptic seizures (PNES). Prolonged PNES (pPNES) are reported by $78 \%$ of patients with PNES and lead to emergency admissions to the intensive care unit (ICU) in $18 \%-27 \%$ of patients. ${ }^{34}$ Acute pharmacological treatment is not indicated as pPNES are usually selflimited and do not respond to medication (beyond the occasional placebo response or deep sedation). pPNES generate no direct secondary physical harm to patients and should be treated with verbal and behavioural interventions only.

As clinical differentiation between epileptic seizures and PNES requires specific semiological expertise, and the treatment imperative for presumed status epilepticus demands almost immediate decision-making, misdiagnosis is common in emergency settings and pPNES are often treated mistakenly with non-indicated medication. Retrospective reviews suggest that patients with misdiagnosed pPNES often receive benzodiazepines (at even higher doses than patients with status epilepticus) $)^{5}$ and frequently require endotracheal intubation as a consequence. ${ }^{6}$ Of note, pPNES are the foremost determinant of healthcare utilisation costs in patients with PNES. ${ }^{47}$

A systematic look at treatment trials for status epilepticus presenting to emergency services (table 1, online supplemental figure 1) reveals that misdiagnosis of pPNES as status epilepticus occurs in various settings and at all levels of treatment: prehospital and in the emergency department; and first-line and second-line therapy. Misdiagnosis is common in both adult and paediatric populations and in both paramedic-led and physician-led emergency services. ${ }^{8-17}$ Remarkably, none of the listed trials had specific procedures in place to recognise and exclude pPNES from study enrolment, nor did they specify protocols for treatment reallocation in cases of misdiagnosis.

To better understand the epidemiology of pPNES misdiagnosed as status epilepticus as well as the risks of harm from non-indicated treatment, we performed an individual patient data analysis from the two largest status epilepticus treatment trials to date: the Rapid Anticonvulsant Medication Prior to Arrival Trial (RAMPART) ${ }^{10}$ and the Established Status Epilepticus Treatment Trial (ESETT) ${ }^{16}{ }^{17}$ We then discuss strategies that may improve recognition of pPNES within emergency medical services 


\begin{tabular}{|c|c|c|c|c|c|c|}
\hline Study & Centres $(n)$, country & Age group & Initial diagnosis made by & Sample size & Rate of misdiagnosis (\%) & $\begin{array}{l}\text { Proportion of } \\
\text { misdiagnoses } \\
\text { specified as } \\
\text { pPNES }\end{array}$ \\
\hline Leppik et $a l^{8}$ & 3, USA & Adults & Physicians, hospital-based & 78 & 13.5 & $1 / 11$ \\
\hline Alldredge et $a l^{9}$ & 10, USA & Adults & Paramedics & 205 & 5 & $10 / 10$ \\
\hline Silbergleit et $a l^{10 *}$ & 79 , USA & Both (children $\geq 40 \mathrm{~kg}$ ) & Paramedics & 893 & 7.1 & $63 / 63$ \\
\hline Chamberlain et al ${ }^{11}$ & 11, USA & $\begin{array}{l}\text { Paediatric ( } 3 \text { months-18 } \\
\text { years) }\end{array}$ & Physicians, hospital-based & 310 & 10 & ns \\
\hline Mundlamuri et al/2 & 1, India & Both (15-65 years) & Physicians, hospital-based & 150 & 0.05 & $1 / 1$ \\
\hline Sánchez Fernández et a $\left.\right|^{13}$ & 9, USA & $\begin{array}{l}\text { Paediatric (1 month-21 } \\
\text { years) }\end{array}$ & Paramedics & 81 & 0 & - \\
\hline Welch et $a l^{14 *}$ & 79, USA & $\begin{array}{l}\text { Paediatric } \\
\text { (weight } \geq 13 \mathrm{~kg} \text { ) }\end{array}$ & Paramedics & 120 & 8.3 & $8 / 10$ \\
\hline Navarro et $a l^{15}$ & 39 , France & Adults & Physicians, prehospital & 136 & 4.4 & $6 / 6$ \\
\hline Kapur et $a l^{16} \dagger$ & 57 , USA & Both ( $\geq 2$ years) & Physicians, hospital-based & 384 & 9.6 & $37 / 37$ \\
\hline Chamberlain et $a l^{17} \dagger$ & 58, USA & Both ( $\geq 2$ years) & Physicians, hospital-based & 462 & nsł & nsł \\
\hline
\end{tabular}

See online supplemental material for search strategy and flow diagram of literature review.

*Publication part of RAMPART.

†Publication part of ESETT.

¥See the Results section for the results of individual patient data analysis.

ESETT, Established Status Epilepticus Treatment Trial; ns, not specified; pPNES, prolonged psychogenic non-epileptic seizures; RAMPART, Rapid Anticonvulsant Medication Prior to Arrival Trial.

and offer recommendations to improve future status epilepticus response protocols in clinical practice and treatment trials.

\section{METHODS}

We analysed available primary data from the two largest status epilepticus treatment trials to date. RAMPART (ClinicalTrials. gov identifier: NCT00809146) is a randomised, double-blind trial comparing intramuscular versus intravenous benzodiazepine as first-line therapy for status epilepticus, which comprised 1023 cases from 893 patients enrolled between June 2009 and January 2011. After excluding cases with eligibility violations, incorrect study medication/dose assignment, repeated enrolment, etc (see original publication for details), 732 patients were included in the final per-protocol analysis. In addition, we analysed data of ESETT (ClinicalTrials.gov identifier: NCT01960075), which comprises 478 cases from 462 patients enrolled between November 2015 and December 2018 at 58 hospital emergency departments across the USA. ${ }^{16}{ }^{17}$ ESETT is a randomised, double-blind study to compare the effectiveness of levetiracetam, fosphenytoin and valproate in patients with established status epilepticus unresponsive to treatment with benzodiazepines. Thus, we base our analysis on data from combined 1210 individual patients. We focus on patients who were discharged with a final diagnosis of non-epileptic seizures. The study protocols of RAMPART and ESETT provided no formal definition or diagnostic criteria (including Electoencephalogram [EEG] requirements) regarding this final diagnosis. We limited our analysis to trial participants aged eight years or older, as PNES can rarely be diagnosed in children younger than this. ${ }^{8-20}$ To assess the relative rate of misdiagnosis, patients' age was stratified into six age brackets: children (8-14 years), adolescents and young adults (15-29 years), younger middle-aged adults (30-44 years), older middle-aged adults ( $45-59$ years), old adults (60-74 years) and very old adults ( $\geq 75$ years).

Within these trials, adverse events were graded according to the National Institute of Health's (NIH) Common Terminology Criteria for Adverse Events and categorised according to the Medical Dictionary for Regulatory Activities classification. $\chi^{2}$ test and Fisher's exact test were used for between-group analyses. The level of significance was $\mathrm{p}<0.05$.

\section{RESULTS}

Among 1210 patients diagnosed and treated as status epilepticus, 980 were aged eight years or older. Of these patients, 79 (8.1\%) were discharged with a final diagnosis of pPNES. Of these, 55 $(70 \%)$ were female and the mean age was 32.6 years (SD: 15.9 years), ranging from eight to 84 years.

Compared with patients with status epilepticus, the relative prevalence of pPNES across age groups was significantly different $\left(\chi^{2}(10)=66.838, p<0.001\right)$, indicating a significant association between age and diagnosis (figure 1). The relative prevalence of pPNES was highest among adolescents and younger adults aged $15-29$ years, with $20.1 \%$ of these patients (38 of 189) eventually recognised as having had pPNES.

Patients with pPNES were misdiagnosed and mistakenly treated for status epilepticus at 39 different study centres, confirming that this is a widespread phenomenon.

\section{First-line treatment}

Regarding the effects of first-line treatment with benzodiazepines, the 46 patients with pPNES from the RAMPART data were analysed. In $14(30 \%)$ patients, prehospital study medication (10 mg intramuscular midazolam plus intravenous placebo, or $4 \mathrm{mg}$ intravenous lorazepam plus intramuscular placebo) was not followed by seizure termination within $10 \mathrm{~min}$ or before arrival at the hospital (primary endpoint). On hospital arrival, seven $(15 \%)$ patients were directly admitted to the ICU, while twelve (26\%) patients were admitted to a non-ICU unit and 27 (59\%) patients were discharged directly from the emergency department. Those admitted to the ICU remained there for a median of one day (range 1-7 days). Overall, the hospital stay of admitted patients had a median duration of three days (range 1-7 days).

In twelve (26\%) patients, a total of 17 adverse events were recorded (online supplemental table S1 presents an overview of all adverse events and their grading across RAMPART and ESETT patients). One of these events-a case of respiratory depression - was reported as being possibly related to the study intervention and led to intubation. 


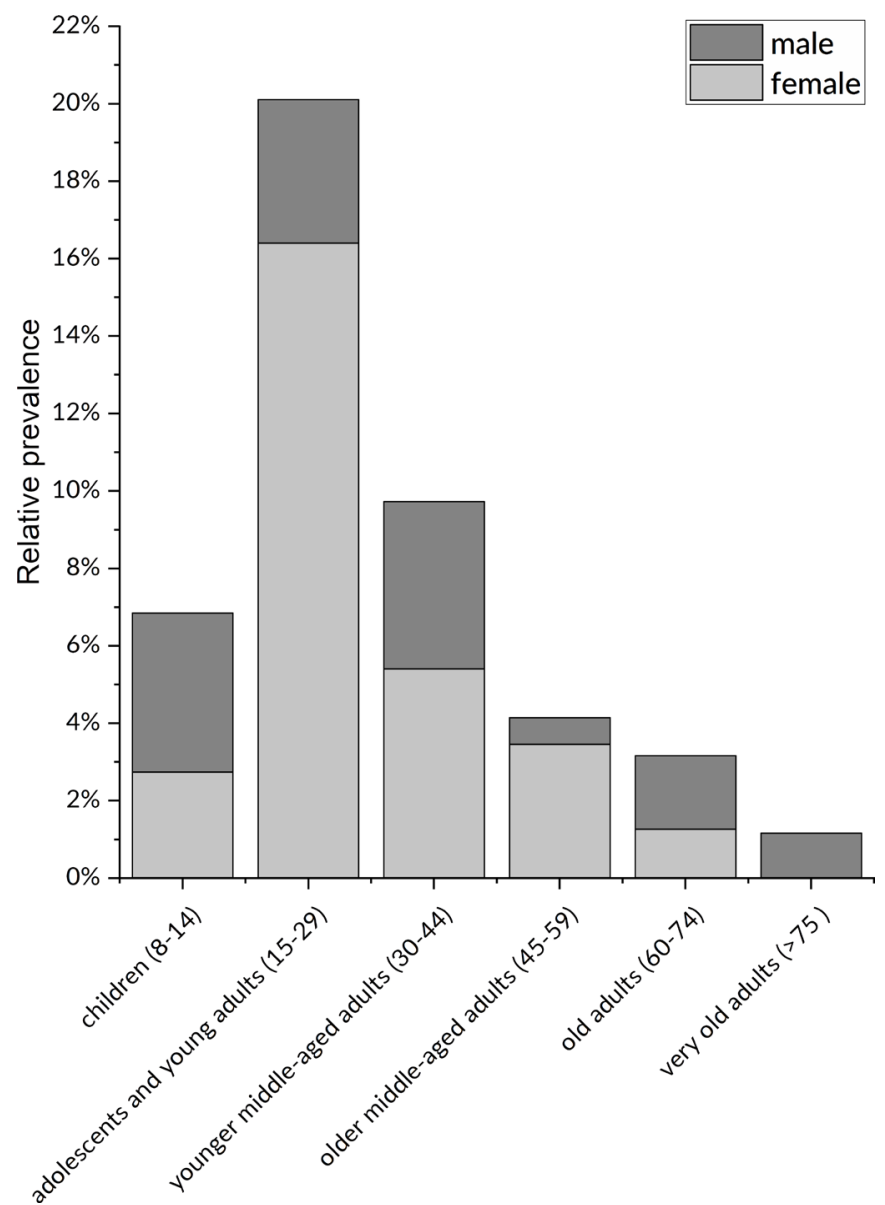

Figure 1 Relative prevalence of prolonged psychogenic non-epileptic seizures in patients overall, by age group and gender.

\section{Second-line treatment}

Cumulative effects of first-line and second-line treatment (levetiracetam, fosphenytoin and valproate) were assessed using data from 33 patients with pPNES unresponsive to antecedent firstline benzodiazepine treatment within ESETT.

Of the 28 patients for whom information on timing of firstline medication was available, $18(55 \%)$ received benzodiazepines before arriving at the emergency department and the rest were medicated in-hospital. Ten patients (31\%) were admitted to the ICU and remained there for up to twelve days (median: one day). Twelve patients (36\%) were admitted to a non-ICU unit, and eleven (33\%) were discharged directly from the emergency department. Overall, the hospital stay of admitted patients had a median duration of one day (range 1-12 days).

All patients were assessed for seizure manifestations and responsiveness at 20 and at $60 \mathrm{~min}$ after the start of the study drug administration. At 20 min assessment, clinically apparent seizures were observed in nine (27\%) patients; at that time, responsiveness to verbal or noxious stimuli was not improved in eleven (33\%) patients. At the 60 min assessment, three patients still had clinically apparent seizures (9\%), while responsiveness was still not improved in nine patients (27\%). The Richmond Agitation Sedation Scale was administered at the 60 min assessment (table 2). Nine patients (27\%) were deeply sedated or unarousable at this point.

In eleven (33\%) patients, a total of 13 adverse events were recorded (online supplemental table S1), four of which were rated as 'serious'. Four events were reported as being likely
Table 2 Richmond Agitation Sedation Scale (RASS) score of patients with pPNES from ESETT at 60 min assessment

\begin{tabular}{llc}
\hline RASS score & Cases & $\%$ \\
\hline+1 restless & 3 & 9.1 \\
0 alert & 4 & 12.1 \\
-1 drowsy & 7 & 21.2 \\
-2 light sedation & 7 & 21.2 \\
-3 moderate sedation & 3 & 9.1 \\
-4 deep sedation & 6 & 18.2 \\
-5 unarousable & 3 & 9.1 \\
\hline
\end{tabular}

ESETT, Established Status Epilepticus Treatment Trial; pPNES, prolonged psychogenic nonepileptic seizures.

related to the study intervention: abdominal pain, hypersensitivity, hypotension and pruritus. The adverse events were graded as more severe in patients who received benzodiazepines before emergency department arrival than those who did not (Fisher's exact test $=10.455, p=0.033$ ). In two patients $(6 \%)$, endotracheal intubation was performed. The adverse events leading to intubation were documented as 'depressed level of consciousness' in one case and 'cerebrovascular accident' in the other case.

\section{DISCUSSION}

In this individual patient data analysis from the two largest status epilepticus trials to date, we found that among patients aged 8 or older the overall rate of misdiagnosed pPNES was $8.1 \%$ and up to $20.1 \%$ in adolescents and young adults. This age group also showed the typical female preponderance for PNES, while gender distribution tended to equalise in children and older adults, in line with previous observations. ${ }^{21} 22$ The annual incidence of confirmed status epilepticus presenting to the emergency department is reported as 16 per $100000 .{ }^{23}$ Based on our analysis, the extrapolated incidence of misdiagnosed pPNES receiving non-indicated medication can be conservatively estimated at around 1.3 per 100000 inhabitants per year, which is equal to that of cerebral venous and sinus thrombosis in the general population. ${ }^{24}$

As treatment is required to commence as soon as $5 \mathrm{~min}$ after seizure onset, a certain degree of aetiology-agnostic emergency decision-making can be encountered in response algorithms for ongoing seizures (i.e., 'shoot first, ask questions later'). ${ }^{25}$ Two common misconceptions might contribute to this approach: (1) benzodiazepines will also be helpful for PNES, and (2) potential adverse effects of benzodiazepines and further escalation of treatment are negligible. Our analysis clearly disproves both of these assumptions. Benzodiazepine treatment does not reliably terminate seizure activity, as 30\% of RAMPART patients with pPNES had ongoing seizures after receiving the study medication. Adverse effects in RAMPART were reported in $26 \%$ of patients with pPNES, including respiratory depression requiring intubation. By design, all participants in ESETT had ongoing seizures despite substantial doses of benzodiazepines at the time of study enrolment, further demonstrating that benzodiazepines should not be expected to effectively halt PNES. Moreover, 27\% of patients with pPNES in ESETT remained deeply sedated at 1 hour after second-line treatment. Although benzodiazepines are not known to cause central apnoea, they depress genioglossus activity and can lead to obstructive apnoea requiring intubation. ${ }^{26}$ Obesity-related obstructive breathing disorders are common in patients with PNES, ${ }^{27}$ so this poses a particular danger in this patient group. 
Admission to the ICU was necessary in $15 \%$ of patients with pPNES in RAMPART and twice that rate in ESETT (31\%). The experience of being treated in an ICU can be highly distressing in itself and can lead to ICU-related post-traumatic stress disorder at a significant rate. Known risk factors for this are premorbid depression, negative emotionality, treatment-seeking behaviours and benzodiazepine sedation, ${ }^{28}$ revealing a particularly dangerous constellation for patients with pPNES. ${ }^{29}$ Lastly, some patients with pPNES were eventually intubated in both trials, an ultimate treatment escalation which has been reported at high rates previously ${ }^{6}$ and harbours well-known risks of its own. ${ }^{30}$

An interesting observation regarding the momentum of the status epilepticus treatment imperative could be made from the ESETT data: patients who were treated with benzodiazepines before arrival at the emergency department had higher rates of unresponsiveness and severe adverse effects in the course of treatment and were more likely to be intubated than those treated in-hospital only. This could be explained by more severe seizure manifestations at onset, but alternatively prehospital interventions might accelerate the momentum of treatment, leading to higher medication doses overall and lowering the threshold for treatment escalation. It is worth noting that invasive overtreatment of pPNES can be potentially lethal. ${ }^{31}$ In a recent cohort study, patients with video EEG-confirmed PNES (and no sign of comorbid epilepsy) had a more than doubled standardised mortality ratio, and the cause of death was documented as 'epilepsy' in 13 of 55 cases (24\%), suggesting the possibility of misdiagnosis and associated iatrogenic harm. ${ }^{32}$ Therefore, the trade-off between early and aggressive treatment to terminate persistent epileptic seizure activity and investing time to rule out or confirm the possibility of pPNES to avoid iatrogenic harm must be weighed carefully.

Another factor underlying the 'shoot first' mentality of convulsive status treatment is the purported lack of reliability of semiological seizure diagnosis. Indeed, semiology alone can be insufficient for a firm diagnosis for many types of seizures and seizure-like events. However, suspected convulsive status epilepticus has pronounced motor symptoms, allowing for a confident diagnosis on clinical grounds in most cases. A recent study on smartphone video recordings of seizures found that convulsive PNES can be identified with $96 \%$ accuracy on video. ${ }^{33}$ Moreover, examination of ongoing seizures allows for quick assessment of interactive responsivity (e.g., resistance to eye-opening, preservation of pupillary and eyelash reflexes, change in seizure intensity in response to others), which can offer near-absolute specificity for PNES. ${ }^{34} 35$ Filming an ongoing seizure for later specialist evaluation should be encouraged as a critical diagnostic intervention and can be justified as such even without prior consent. ${ }^{2}$ Paramedics, emergency department staff, neurologists, and intensivists should be sensitised regarding the relative incidence of PNES and trained in semiological recognition of seizure types to reduce the rate of misdiagnosis and the risk of harm from overtreatment. The importance of obtaining ictal video, early EEG and postictal laboratory markers such as prolactin and creatine kinase should inform emergency work-up algorithms to reduce misdiagnosis.

The observed rates of pPNES misdiagnosis and risks of harm from non-indicated treatment should give cause to reconsider status epilepticus response protocols, especially in clinical trials. Highly specific semiological indicators of pPNES should be considered at enrolment and pPNES should be explicitly listed as exclusion criteria. Clinical reassessment, possibly operationalised through semiological checklists, should further be required at every step of treatment escalation. As misdiagnosed
pPNES can be expected at a rate of 5\%-10\%, specific protocols for de-escalation and reallocation of treatment should ideally be provided to trialists. Lastly, study analysis plans should include separate epidemiological and safety subanalyses for patients later diagnosed with pPNES.

Contributors JJ and SP formulated the initial study idea. RM contributed to refinement of the structure. RM managed the systematic literature review. JJ performed the data preparation and data analysis. SP and RM advised on evaluation. SP formulated the discussion. All authors contributed to the discussion, writing the manuscript and reviewed the final manuscript.

Funding The authors have not declared a specific grant for this research from any funding agency in the public, commercial or not-for-profit sectors.

Competing interests SP receives royalties from Springer for a book on functional neurological disorders. The other authors declare no conflicts of interest.

\section{Patient consent for publication Not required.}

Provenance and peer review Not commissioned; externally peer reviewed.

Data availability statement Data are available upon reasonable request. This research is based on the National Institute of Neurological Disease and Stroke's Archived Clinical Research data of RAMPART ('Rapid Anticonvulsant Medication Prior to Arrival Trial', Pls: Silbergleit, R, Lowenstein, DH and Durkalski, VL) and ESETT ('A Multicenter, Randomized, Blinded, Comparative Effectiveness Study of Fosphenytoin, Valproic Acid, or Levetiracetam in the Emergency Department Treatment of Patients With Benzodiazepine-refractory Status Epilepticus', Pls: Silbergleit R, Chamberlain $J$ and Elm J) received from the Archived Clinical Research Dataset website (https:// www.ninds.nih.gov/Current-Research/Research-Funded-NINDS/Clinical-Research/ Archived-Clinical-Research-Datasets).

Supplemental material This content has been supplied by the author(s). It has not been vetted by BMJ Publishing Group Limited (BMJ) and may not have been peer-reviewed. Any opinions or recommendations discussed are solely those of the author(s) and are not endorsed by BMJ. BMJ disclaims all liability and responsibility arising from any reliance placed on the content. Where the content includes any translated material, BMJ does not warrant the accuracy and reliability of the translations (including but not limited to local regulations, clinical guidelines, terminology, drug names and drug dosages), and is not responsible for any error and/or omissions arising from translation and adaptation or otherwise.

Open access This is an open access article distributed in accordance with the Creative Commons Attribution Non Commercial (CC BY-NC 4.0) license, which permits others to distribute, remix, adapt, build upon this work non-commercially, and license their derivative works on different terms, provided the original work is properly cited, appropriate credit is given, any changes made indicated, and the use is non-commercial. See: http://creativecommons.org/licenses/by-nc/4.0/.

\section{ORCID iDs}

Johannes Jungilligens http://orcid.org/0000-0002-4846-8337

Rosa Michaelis http://orcid.org/0000-0002-2577-0824

Stoyan Popkirov http://orcid.org/0000-0001-6168-0036

\section{REFERENCES}

1 Trinka E, Cock H, Hesdorffer D, et al. A definition and classification of status epilepticus--Report of the ILAE Task Force on Classification of Status Epilepticus. Epilepsia 2015;56:1515-23

2 Crawshaw AA, Cock HR. Medical management of status epilepticus: emergency room to intensive care unit. Seizure 2020;75:145-52.

3 Reuber M, Pukrop R, Mitchell AJ, et al. Clinical significance of recurrent psychogenic nonepileptic seizure status. J Neurol 2003;250:1355-62.

4 Seneviratne U, Low ZM, Low ZX, et al. Medical health care utilization cost of patients presenting with psychogenic nonepileptic seizures. Epilepsia 2019;60:349-57.

5 Holtkamp M, Othman J, Buchheim K, et al. Diagnosis of psychogenic nonepileptic status epilepticus in the emergency setting. Neurology 2006;66:1727-9.

6 Viarasilpa T, Panyavachiraporn N, Osman G, et al. Intubation for psychogenic non-epileptic attacks: frequency, risk factors, and impact on outcome. Seizure 2020;76:17-21.

7 Seneviratne U, Minato E, Paul E. How reliable is ictal duration to differentiate psychogenic nonepileptic seizures from epileptic seizures? Epilepsy Behav 2017;66:127-31

8 Leppik IE, Derivan AT, Homan RW, Leppik Ilo E, et al. Double-Blind study of lorazepam and diazepam in status epilepticus. JAMA 1983;249:1452.

9 Alldredge BK, Gelb AM, Isaacs SM, et al. A comparison of lorazepam, diazepam, and placebo for the treatment of out-of-hospital status epilepticus. N Eng/ J Med 2001;345:631-7

10 Silbergleit R, Durkalski V, Lowenstein D, et al. Intramuscular versus intravenous therapy for prehospital status epilepticus. N Engl J Med 2012;366:591-600. 
11 Chamberlain JM, Okada P, Holsti M, et al. Lorazepam vs diazepam for pediatric status epilepticus. JAMA 2014;311:1652.

12 Mundlamuri RC, Sinha S, Subbakrishna DK, et al. Management of generalised convulsive status epilepticus (SE): A prospective randomised controlled study of combined treatment with intravenous lorazepam with either phenytoin, sodium valproate or levetiracetam--Pilot study. Epilepsy Res 2015;114:52-8.

13 Sánchez Fernández I, Abend NS, Agadi S, et al. Time from convulsive status epilepticus onset to anticonvulsant administration in children. Neurology 2015:84:2304-11.

14 Welch RD, Nicholas K, Durkalski-Mauldin VL, et al. Intramuscular midazolam versus intravenous lorazepam for the prehospital treatment of status epilepticus in the pediatric population. Epilepsia 2015;56:254-62

15 Navarro V, Dagron C, Elie C, et al. Prehospital treatment with levetiracetam plus clonazepam or placebo plus clonazepam in status epilepticus (SAMUKeppra): a randomised, double-blind, phase 3 trial. Lancet Neurol 2016;15:47-55.

16 Kapur J, Elm J, Chamberlain JM, et al. Randomized trial of three anticonvulsant medications for status epilepticus. N Engl J Med 2019;381:2103-13.

17 Chamberlain JM, Kapur J, Shinnar S, et al. Efficacy of levetiracetam, fosphenytoin and valproate for established status epilepticus by age group (ESETT): a double-blind responsive-adaptive, randomised controlled trial. Lancet 2020;395:1217-24.

18 Plioplys S, Doss J, Siddarth P, et al. A multisite controlled study of risk factors in pediatric psychogenic nonepileptic seizures. Epilepsia 2014;55:1739-47.

19 Doss J, Caplan R, Siddarth P, et al. Risk factors for learning problems in youth with psychogenic non-epileptic seizures. Epilepsy Behav 2017;70:135-9.

20 Kozlowska K, Chudleigh C, Cruz C, et al. Psychogenic non-epileptic seizures in children and adolescents: Part I - Diagnostic formulations. Clin Child Psychol Psychiatry 2018;23:140-59.

21 Duncan R, Oto M, Martin E, et al. Late onset psychogenic nonepileptic attacks. Neurology 2006:66:1644-7.

22 Operto FF, Coppola G, Mazza R, et al. Psychogenic nonepileptic seizures in pediatric population: a review. Brain Behav 2019;9:e01406.
23 Nazerian P, Lazzeretti D, Vanni S, et al. Incidence, management and short-term prognosis of status epilepticus in the emergency department: a population survey. Eur J Emerg Med 2019;26:228-30.

24 Weimar C. Diagnosis and treatment of cerebral venous and sinus thrombosis. Curr Neurol Neurosci Rep 2014;14:417.

25 Gloss D, Weisberg L. Neurology for the specialty boards. Lippincott Williams \& Wilkin, 2006.

26 Gueye PN, Lofaso F, Borron SW, et al. Mechanism of respiratory insufficiency in pure or mixed drug-induced coma involving benzodiazepines. J Toxicol Clin Toxicol 2002;40:35-47.

27 Popkirov S, Stone J, Derry CP. Abnormal sleep in patients with epileptic or dissociative (non-epileptic) seizures: a polysomnography study. Eur J Neurol 2019;26:255-60.

28 Marra A, Pandharipande PP, Patel MB. Intensive care unit delirium and intensive care Unit-Related posttraumatic stress disorder. Surg Clin North Am 2017;97:1215-35.

29 Popkirov S, Asadi-Pooya AA, Duncan R, et al. The aetiology of psychogenic nonepileptic seizures: risk factors and comorbidities. Epileptic Disord 2019;21:529-47.

30 Cook T, Behringer EC, Benger J. Airway management outside the operating room. Curr Opin Anaesthesiol 2012;25:461-9.

31 Reuber M, Baker GA, Gill R, et al. Failure to recognize psychogenic nonepileptic seizures may cause death. Neurology 2004;62:834-5.

32 Nightscales R, McCartney L, Auvrez C, et al. Mortality in patients with psychogenic nonepileptic seizures. Neurology 2020;95:e643-52.

33 Tatum WO, Hirsch LJ, Gelfand MA, et al. Assessment of the predictive value of outpatient smartphone Videos for diagnosis of epileptic seizures. JAMA Neurol 2020;77:593.

34 Syed TU, LaFrance WC, Kahriman ES, et al. Can semiology predict psychogenic nonepileptic seizures? A prospective study. Ann Neurol 2011;69:997-1004.

35 Wardrope A, Wong S, McLaughlan J, et al. Peri-Ictal responsiveness to the socia environment is greater in psychogenic nonepileptic than epileptic seizures. Epilepsia 2020:61:758-65 